Note: this is a post-print draft of the journal article:

Dickinson, A.S., Taylor, A.C., Jeffers, J.R.T, Browne, M. (2010) "Performance of the Resurfaced Hip: Part 2- The Influence of Prosthesis Stem Design on Remodelling and Fracture of the Femoral Neck". Proceedings of the Institution of Mechanical Engineers Part H: Journal of Engineering in Medicine 224: 841-851

The final, fully proofed and peer-reviewed journal article is available from the publisher online, via the following link:

http://pih.sagepub.com/content/224/7/841.abstract 


\title{
Performance of the Resurfaced Hip: Part 2- The Influence of Prosthesis Stem Design on Remodelling and Fracture of the Femoral Neck
}

\author{
A S Dickinson ${ }^{1}$, A C Taylor ${ }^{2}$, J R T Jeffers ${ }^{2}$, M Browne ${ }^{1}$ \\ ${ }^{1}$ Bioengineering Science Research Group, University of Southampton, Southampton, UK \\ ${ }^{2}$ Finsbury Development Ltd, Leatherhead, Surrey, UK
}

\begin{abstract}
Hip Resurfacing is a popular treatment for osteoarthritis in young, active patients. Previous studies have shown that occasional failures- femoral neck fracture and implant loosening, possibly associated with bone adaptation- are affected by prosthesis sizing and positioning, in addition to patient and surgical factors.

Aiming to improve tolerance to surgical variation, Finite Element modelling was used to indicate the effects of prosthesis metaphyseal stem design on bone remodelling and femoral neck fracture, with a range of implant orientations.
\end{abstract}

The analysis suggested that the intact femoral neck strength in trauma could be maintained across a wider range of varus-valgus orientations for short-stemmed and stemless prostheses. Furthermore, the extent of periprosthetic bone remodelling was lower for the short-stemmed implant, with slightly reduced stress shielding and considerably reduced densification around the stem, potentially preventing further progressive proximal stress shielding.

The study suggests that a short-stemmed resurfacing head offers improved tolerance to misalignment and remodelling stimulus over traditional designs. Whilst femoral neck fracture and implant loosening are multifactorial, biomechanical factors are of clear importance to the clinical outcome so this may reduce the risk for patients at the edge of the indications for hip resurfacing, or shorten the surgical learning curve.

Keywords: hip resurfacing, finite element analysis, bone remodelling, neck fracture, prosthesis design 


\section{Introduction}

Resurfacing Hip Replacement (RHR) has become established as an alternative to traditional Total Hip Replacement (THR) for young, active patients. However, there are two main early or medium-term mechanical failure modes of the resurfaced femoral head, which have been found to be influenced by the prosthesis surgical positioning. Fracture of the femoral neck is the most common early failure mode, occurring in $0.5-2 \%$ of patients. In mechanical terms, this has been linked to excessive varus prosthesis alignment, notching of the femoral neck, and exposure of reamed cancellous bone due to incomplete seating of the prosthesis. In addition, inadequate supporting bone quality, the presence of cysts and necrotic bone, and microfractures from surgical loads are believed to play a role [1-6].

The second, medium-term failure mode- migration and loosening of the prosthesis head- may be preceded by radiographic changes around the prosthesis, including the formation of radiolucencies and dense 'pedestal lines' around the prosthesis metaphyseal stem, densification of the inferior-medial femoral neck, and narrowing of the femoral neck distal to the prosthesis rim [7-11]. Although they appear to have no definitive link to clinical failures, they are more common in failing hips [7], and the incidence of such changes may be as high as $90 \%$ [11]. Narrowing of the femoral neck may be explained by disruption to the femoral head blood supply, inflammatory response to wear particles and impingement [6, 10-12]. However, it is reported to take place substantially during the first $2-3$ years postoperatively, after which it stabilises up to 7 years $[10,11]$, which, in common with its high incidence, would be consistent with stabilising bone adaptation.

This failure mode may also be linked to prosthesis positioning; previous computational modelling research has shown that if the resurfacing head is oriented in valgus the remodelling stimulus may be sufficient to produce radiographically visible narrowing of the femoral neck [13]. In Part 1 of this study, results of an FE modelling investigation were presented, suggesting that neck narrowing could be caused by the reduction in horizontal femoral offset which could result from valgus positioning, or from undersizing of the femoral head prosthesis. This would reduce the moment arm for the forces on the femoral head and neck. Although a reduced femoral offset would reduce the range of abduction and increase the abductor muscle and joint contact forces somewhat $[14,15]$, medialisation of the joint centre 
as a result of cup positioning- a common outcome of RHR surgery [16]- was predicted to produce a net reduction in the joint contact force. As such, in extreme cases it was predicted that the femoral neck bending moment and therefore the strain energy density in the superior femoral neck could be reduced sufficiently for narrowing to occur by stress shielding.

In addition to implant positioning, there is evidence to suggest that both of the failure modes in question have a link to the presence of the prosthesis metaphyseal stem:

- femoral neck fracture because the stem bore reduces the neck load bearing cross sectional area and its tip acts as a stress concentration if it approaches the surface of the femoral neck, particularly in varus orientation $[3,17]$, and

- $\quad$ prosthesis migration and loosening because they are preceded by radiographically visible bone density adaptations [7], particularly resorption inside the prosthesis shell, and bone densification around the stem and in the medial femoral neck, which may lead to increased proximal stress shielding.

This is the second part of a computational modelling study on the biomechanics of hip resurfacing. Part 1 of this study, reported previously, investigated the effect of the sizing and positioning of a traditional design prosthesis on the risk of femoral neck fracture, and the remodelling stimulus in the supporting bone. As well as highlighting the effects of varus-valgus positioning and reductions in the horizontal femoral offset of the resurfaced hip, the results from Part 1 of this study suggested an influence of the prosthesis stem on these failure modes. First, the models predicted that a strain concentration would be produced in the bore for the implant's metaphyseal stem, particularly in varus orientation when the tip of the stem bore is located near to the surface of the femoral neck. As such, the results suggested that with poor positioning, the presence of a long stem and a bore drilled to accommodate it are partially responsible for femoral neck fractures. Furthermore, the models predicted that bone densification would be stimulated around the stem and its bore, consistent with the previously cited clinical observations and noted to be more common in failing prostheses. Although the stiff shell of the implant is probably the cause of stress shielding inside the femoral head, localised load transfer around the prosthesis stem could lead to further stress shielding in the 
femoral neck, so the prosthesis metaphyseal stem may also be linked to longer term prosthesis loosening. The results predicted a clear link between poor prosthesis positioning and these failure modes, in accordance with clinical observations, but suggested that in the case of poor positioning, the stem may be involved in any subsequent failure.

The design of current resurfacing heads has evolved over several decades since the earliest surface replacements [18], and the bearing surface has been optimised for sizing to typical ranges of patient anatomy, achieving adequate post-operative range of motion and excellent tribological performance; as such, all the available resurfacing prostheses have similar shell designs. However, there is some variety in metaphyseal stem designs including the presence and extent of taper along the stem's length, its surface finish and, indeed, the stem's presence. Some earlier designs have reached long term follow-up without a stem [19-21] and poor results may be explained by aseptic loosening associated with a polyethylene acetabular cup, as seen with all early surface replacements. However, stemless resurfacing heads were still susceptible to neck fracture and prosthesis loosening, and with the results from Part 1 of this study in mind, the influence of the stem design on these failure modes was investigated, with the particular goal of improving the tolerance to misalignment.

\section{Methods and Materials}

A subject specific CAD model of the proximal third of the femur was created using a computer tomography (CT) scan, the full details of which are described in Part 1 of this study. The femoral head was resurfaced with a prosthesis representative of the BHR (Smith\&Nephew, Memphis, TN, USA) and the ADEPT (Finsbury Orthopaedics, Leatherhead, UK) designs (henceforth referred to as the 'traditional design'), implanted with the stem aligned with the femoral neck axis, and with $\pm 10^{\circ}$ varus-valgus orientation. This was the maximum variation which could be achieved with an implant sized to allow recreation of the natural joint centre, without notching the femoral neck or leaving exposed reamed cancellous bone. Two alternative prosthesis designs were modelled, a 'short stemmed design' featuring a cylindrical, non-tapering stem which terminated at the spherical centre of the bearing surface, and a 
'stemless design' featuring no metaphyseal stem. Other than the stem design, the geometry was kept identical to the traditional design. The three prostheses are shown in Fig. 1.

The prostheses were modelled as fixed with an approximately $2.5 \mathrm{~mm}$ thick cement layer (stiffness 2.8GPa [22]) and the stem was located in a parallel sided bore of the same diameter as the stem's cylindrical portion, and over-reamed by $5 \mathrm{~mm}$ in length. The stem-bone interface was simulated in sliding contact with the bone with a nominal friction coefficient of 0.4 [22]

The same three load cases were modelled as in Part 1 of this study, representing one gait and two traumatic scenarios: stumbling in stance, and a sideways fall onto the greater trochanter. The gait load case was simulated to analyse the effect of the prosthesis design on bone remodelling, and the traumatic load cases were used to investigate the femoral neck fracture risk. Again, the femoral neck fracture risk was compared for the three designs, quantified as the load at which bone yield initiated using a risk factor (RF) for each element [23-27], giving yield when RF>1. The distribution of damaged bone elements was also inspected for implanted bones under the load at which damage initiation occurred in the intact femur. The risk factor was calculated as the ratio of the element's highest magnitude principal strain to a yield strain value from in-vitro data [28]. The calculation took into account the element's strain state; tensile or compressive elemental strain and yield strain values were used depending upon whether the first or third principal strain value was larger. Also, the type of bone represented by the element was taken into account, with cancellous or cortical yield strain values used [28] depending upon the element's density. The extent of bone remodelling resulting from implantation was quantified using a strain energy density (SED) stimulus $[29,30]$, whereby the percentage change in SED was calculated from the pre- to post-operative conditions and the volume of remodelling bone was found using a threshold level of stimulus required for bone resorption or densification to occur ( $\pm 75 \%$ for the elderly patient $[13,29,31]$ and $\pm 50 \%$ for a younger patient with a more active metabolism). 


\section{Results}

Results are presented in two sections- considering the femoral neck fracture risk in traumatic loading, and considering the bone remodelling stimulus in gait loading.

\subsection{Femoral Neck Fracture Risk}

The femoral neck fracture load was predicted for the bone resurfaced with the three prosthesis designs in varus, neutral and valgus orientations and compared to the natural bone, for stumbling and sideways falling loads. The results are included in Fig. 2 and Fig. 3, with the distribution of failing elements for a given load: $6 \mathrm{kN}$ in stumbling (Fig. 4) and 3kN in falling (Fig. 5).

As reported in Part 1 of this study, for a femur resurfaced with a traditional design implant, a positive correlation was observed between the load at which damage initiated in the femoral head and neck bone and increasing valgus prosthesis orientation under stumbling loading. The model predicted that the damage initiation load would be decreased by approximately $20 \%$ with varus implant orientation and $9 \%$ in neutral orientation. In valgus orientation, the predicted neck fracture load was within $2 \%$ of the intact case. Inspection of the damage location in Fig. 4 indicates that this may have resulted from the weakening effect of the bore for the prosthesis stem, where damage initiates for the neutral and varus models. In the valgus model, the bore is located closer to the largely compressively loaded medial femoral neck so it has less of a weakening effect, and damage initiation was in a similar location and at a similar load to the intact bone.

Compared to the stemmed, traditional design prosthesis, the short stemmed and stemless designs had less weakening effect upon the femoral neck. For the stemless design in all orientations, the femoral neck strength was predicted to be within $5 \%$ of that for the intact bone, and the strength with the short stemmed design was within $2 \%$ of the intact case (Fig. 2). The damaged bone distribution plots in Fig. 4 suggest that this may be related to the removal of the long stem and its bore as both new designs led to the same bone damage initiation location as the intact bone, in the superior femoral neck.

Fig. 3 shows the damage initiation loads and Fig. 5 the locations for the sideways falling scenario; all fractures were predicted to initiate at the anterior-medial surface of the femoral neck. All designs implanted 
in all orientations were predicted to give a femoral neck damage initiation load within $5 \%$ of the intact case, so the proximity of the stem bore to the stress concentrated medial neck does not appear to weaken the bone.

\subsection{Bone Remodelling Stimulus}

The remodelling stimulus (percentage change in strain energy density) was calculated for the nine implanted cases and is shown for a cross section along the femoral neck axis in Fig. 6. This shows the locations in which bone resorption and densification would be expected; for an elderly patient these correspond to a stimulus below $-75 \%$ and above $75 \%$ respectively, shown by the bottom and top contours on the charts, and for a younger patient a stimulus of $\pm 50 \%$. In all cases, extensive stress shielding was predicted within the superior femoral head. For the traditional design prosthesis, densification was predicted around the stem bore, particularly around the narrowest point of the femoral neck and at the tip of the bore. Use of the stemless and short stemmed prostheses was not predicted to remove the stress shielding inside the femoral head. However, it can be seen on the charts in Fig. 7 (which quantify the extent of stress shielding and hypertrophy for the two threshold stimulus levels) that stress shielding was reduced by $9-12 \%$ for the young patient and $3-10 \%$ in the elderly patient with the short stemmed implant in valgus positioning. Conversely, the extent of hypertrophic bone was predicted to be reduced considerably with the removal of the long stem and its bore, particularly in neutral and valgus orientations, which led to an overall reduction in the volume of remodelling bone of up to $19 \%$ for the elderly and $21 \%$ for the young patient. This was when the prosthesis was in valgus positioning- the worst case for the traditional design.

The stemless head was predicted to stimulate an even lower volume of bone densification as the stem is removed completely. However, retaining the entire internal volume of the femoral head led to the prediction of an increased volume of stress shielded bone, by $10-17 \%$ in the elderly patient and $4-13 \%$ in the young patient. 


\section{Discussion}

Although hip resurfacing surgery has achieved excellent medium term clinical results, there is still an incidence of early femoral neck fractures and longer term femoral prosthesis loosening. In addition to patient selection and education, biomechanical quantities including surgical positioning and prosthesis sizing play a major role in the outcome, as was discussed in Part 1 of this study. Although there is an understanding of optimal prosthesis positioning to avoid these failures, problems such as the learning curve associated with hip resurfacing surgery will continue to make it difficult to achieve the desired prosthesis positioning. Therefore, aspects of the prosthesis geometry were investigated to attempt to find a design which is more tolerant to mal-positioning. The study employed an FE model, and considered the effect of the metaphyseal stem length on the femoral neck fracture risk and the extent and pattern of bone remodelling in the femoral head and neck.

First, the effect of the prosthesis stem length upon the femoral neck strength was investigated, in stumbling and sideways falling events. During stumbling, the long stemmed, traditional design prosthesis reduced the damage initiation load in the femoral neck when implanted with varus orientation by up to $20 \%$ compared to the intact bone. The new designs maintained an intact femoral neck without a bore drilled into it to accommodate the stem. This resulted in very similar damage initiation to the intact bone, in the same location and at a load within $5 \%$ for the stemless design and $2 \%$ for the short stemmed design. The correlation between neck fracture strength and varus-valgus orientation predicted by the model for the traditional implant design is corroborated by clinical evidence in which excessive varus orientation is a risk factor $[3,17]$. Furthermore, it agrees with in-vitro mechanical test results on resurfaced synthetic and cadaveric femurs [32] which observed a similar linear reduction in neck strength with varus orientation, from a recreation of the natural femoral neck's strength when the prosthesis has $10^{\circ}$ of relative valgus orientation. Furthermore, Appleyard et al [33] studied cadaveric femoral neck strain measurements after resurfacing with a cemented stemless shell, followed by addition of attached and detached stems, with and without a bore, and reported that the stemless head gave the closest femoral neck strain pattern to the intact femur in comparison to the traditional long stemmed prosthesis configurations. The model's results suggested that under sideways falling loads, the neck fracture risk was very similar to the intact case for the three prosthesis stem designs in valgus, neutral and varus orientations, with identical damage initiation 
locations at loads within $5 \%$ of the intact case. It can be concluded that in this loading condition, resurfacing with shorter stemmed or stemless prostheses would have no significant effect upon the femoral neck fracture risk.

Reports of the use of stemless resurfacing heads in the past have been positive. Wagner [34] reported upon 426 hips with stemless resurfacing heads at a minimum follow-up of 6 months, an accepted at-risk period for femoral neck fracture [3], and identified only one femoral neck fracture which occurred pathologically, in a patient with considerable cystic degeneration of the femoral neck. In other cohorts, femoral neck fracture has had an incidence of approximately $1-3 \%[19,35,36]$, and in several cases could be explained by surgical technique. Ritter et al [21] reported an $18 \%$ femoral neck fracture rate, but occurring at 6 months to nineteen years follow-up and an average of eight years, indicating a different mode of failure from current designs, possibly related to progressive bone adaptation. They reported extensive narrowing of the femoral neck in their cohort, which was suggested in Part 1 of this study to be highly influenced by prosthesis positioning and sizing. Comparing the results of both parts of this study, radiographic changes at the surface of the femoral neck potentially leading to neck narrowing were only predicted to occur in extremes of prosthesis position and sizing, as reported in Part 1, so these variables may be predicted to have a greater influence than the prosthesis design variables considered. With the surgeries reported in these clinical studies taking place between 1974 and 1984 the results are more convincing still, considering that they were undertaken without today's advanced instrumentation or full understanding of optimal positioning and patient selection, and largely within the surgeons' learning period of their first 100 operations. Therefore, this modelling study's predictions for reduced short term femoral neck fracture risk for the short stemmed and stemless designs are consistent with previous clinical observations.

The second part of the study looked at the effects of prosthesis metaphyseal stem design upon remodelling in the periprosthetic bone. The results of the traditional design implant were discussed fully in Part 1 of this study, with patterns of immediate postoperative remodelling stimulus representative of clinically observed radiographic changes and in agreement with previous modelling studies: resorption 
inside the superior femoral head, and densification around the metaphyseal stem to form sclerotic 'pedestal lines' $[7,13,17,22,31,37-46]$. The results of this study suggested that the femoral head resorption was caused largely by stress shielding from the thick metal shell of the prosthesis head, and was only slightly improved in the immediate postoperative situation by the stem design. Shortening or removal of the stem would prevent its supposed distal load transfer, and was seen to remove the considerable region of densifying bone around the stem that supports the traditional prosthesis and the bore drilled to accommodate it. In addition to instantaneous effects, this may also be beneficial by avoiding progressive bone resorption, which could occur for traditional designs where load transfer by the stem could increase over time as the bone densifies around it and in the medial neck. The greatest improvement was observed for the short stemmed design in valgus orientation, which was the worst case for the traditional design prosthesis. The results indicated that retaining a short stem would be preferable to removing it completely in terms of the strain distribution inside the femoral head, in addition to its role in aiding the surgeon with introducing the head at the correct angle. Despite its shorter length than traditional designs, the stem could still have this function provided it contacts the central bore before the tapered cylindrical face inside the prosthesis meets the shoulder on the cylindrically cut femoral head.

Ritter et al's results [21] showed that all of their eight late femoral neck fractures and femoral loosenings had progressively narrowed femoral necks despite a stemless prosthesis (the Indiana conservative, DePuy Orthopaedics, Inc., USA), but Pollard et al [7] identified that loosening of contemporary stemmed resurfacing heads was most likely in patients with a combination of the listed radiographic changes. This clinical data supports this study's prediction of reduced bone remodelling and potentially a lower loosening rate as a result of shortening the resurfacing head's metaphyseal stem.

As reported in Part 1 of this study, there are several additional potential causes of radiographic changes around resurfacing head prostheses and their loosening. Other previously suggested causes include inflammatory response to wear particles, impingement and bone necrosis, possibly caused by exothermic bone cement polymerisation or disruption of the blood supply to the femoral head $[6,10-12]$. The progressive nature of the radiographic changes observed around hip resurfacing prostheses which may stabilise with time $[10,11]$ may be indicative that bone remodelling plays a considerable part, but the high incidence of radiographic changes implies that some combination of these effects is responsible. The results 
of this study suggest that the bone remodelling effect at least may be reduced by shortening the prosthesis metaphyseal stem, which could also improve its tolerance to misalignment in terms of the femoral neck fracture risk. Any such improvements would have potential benefits for the patient at the edge of the indications for hip resurfacing, and to shorten the surgeon learning curve.

As with all computational modelling studies, the results are subject to the limitations of the modelling techniques and the simplifications made in the model pre-processing. These are discussed in full in Part 1 of this study, but summarised here. Verification checks were conducted including a mesh convergence analysis and comparison of the model's displacements and strains to other modelling predictions and clinical measurements. Furthermore the modelling process was simplified in terms of the use of a single femur model from an orthopaedic disease-free patient, idealised prosthesis positioning and cement penetration, the use of the same pre- and post-operative loading conditions and of a single load case for each loading scenario. The traumatic load cases were chosen because they represented the worst cases of in-vitro testing, and the gait load case for bone remodelling prediction because it represents the great majority of daily activity. These simplifications had to be made in order to avoid confounding variables, and to isolate the effects of the prosthesis design, which was judged not to have a considerable effect upon them. With identical prosthesis positioning, the investigated design variables will have no effect upon the joint contact and muscle forces. Furthermore, the effects of osteoarthritis- limited to an extent such that hip resurfacing is still a suitable treatment- are likely to be greatest in the subchondral bone which is removed by the surgical cuts, so since the design variables considered are restricted to the stem, a CT scan from a disease-free patient can be used. Corroborating evidence between the model's predictions and clinical observations has been cited where possible, in order to give confidence in the conclusions drawn. Ultimately, the use of such computational modelling results is strongest when they are comparative, and that is the basis of the approach taken in this study. 


\section{Conclusions}

In the young, active osteoarthritis patient, an excellent outcome is possible with hip resurfacing. Whilst the importance of the positioning and sizing of prosthesis designs in current clinical use has been established, this modelling study aimed to identify the role of prosthesis metaphyseal stem design upon the two main femoral failure modes (neck fracture and prosthesis loosening), with a view to improving tolerance to malpositioning. The results indicate the following:

1) The presence of a bore for a long metaphyseal stem has a stress concentrating effect in the femoral neck, which reduces its strength should it approach the stressed surface of the bone. A considerable amount of bone remodelling is also predicted to occur around the stem.

2) Shortening or removing the metaphyseal stem maintains the intact femoral neck strength under stumbling loads across a wider range of prosthesis orientations, because no bone is removed from the femoral neck. As such, the tolerance to poor implant positioning would be improved.

3) The extent of bone remodelling around a short stemmed resurfacing head is lower. This can reduce femoral head stress shielding slightly, but in particular prevent femoral neck bone densification around the stem of a traditional prosthesis, which could reduce further progressive proximal stress shielding.

As such, the models in this study have proven capable of predicting the biomechanical behaviour of the resurfaced femoral head as identified clinically and in other computational studies. In addition the models have enabled predictions to be made regarding the effect of the design of the prosthesis metaphyseal stem, indicating that a shorter stem may reduce the incidence both of femoral neck fracture and of adverse bone remodelling. Hip resurfacing provides an excellent solution for the young, male patient, but there is scope to reduce the risks of femoral neck fracture and loosening of the femoral prosthesis in a broader patient cohort; this study predicts that a shorter stemmed prosthesis could achieve this aim. 


\section{ACKNOWLEDGMENT}

This study was funded by a collaborative Knowledge Transfer Partnership between Finsbury Development

Ltd and the UK Government Technology Strategy Board, undertaken at the University of Southampton, with the former having input in the study design. 


\section{REFERENCES}

1. Amstutz, H.C., Campbell, P A, Le Duff, M J, Fracture of the neck of the femur after surface arthroplasty of the hip. J Bone Joint Surg [Am], 2004. 86: p. 1874-1877.

2. Little, C.P., Ruiz, A L, Harding, I J, McLardy-Smith, P, Gundle, R, Murray, D W, Athansou, N A, Osteonecrosis in retrieved femoral heads after failed resurfacing arthroplasty of the hip. J Bone Joint Surg [Br], 2005. 87-B: p. 320-323.

3. Shimmin, A.J., Back, D, Femoral Neck Fractures Following Birmingham Hip Resurfacing: A National Review of 50 Cases. J Bone Joint Surg [Br], 2005. 87-B: p. 463-4.

4. Siebel, T., Maubach, S, Morlock, M M, Lessons learned from early clinical experience and results of 300 ASR hip resurfacing implantations. Proc IMechE H, 2006. 220: p. 345353.

5. Treacy, R.B.C., McBryde, Pynsent,, Birmingham hip resurfacing arthroplasty. J Bone Joint Surg [Br], 2005. 87-B: p. 167-170.

6. Beaulé, P.E., Campbell, P A, Hoke, R, Dorey, F, Notching of the femoral neck during resurfacing arthroplasty of the hip. A vascular study. J Bone Joint Surg [Br], 2006. 88-B: p. 35-39.

7. Pollard, T.C.B., Baker, R P, Eastaugh-Waring, S J, Bannister, G C, Treatment of the Young Active Patient with Osteoarthritis of the Hip: A Five- to Seven-Year Comparison of Hybrid Total Hip Arthroplasty and Metal-on-Metal Resurfacing. J Bone Joint Surg [Br], 2006. 88-B: p. 592-600.

8. McMinn, D., Treacy, R, Lyn, K, Pynsent, P, Metal-on-metal surface replacement of the hip. Clin Ortho Rel Res, 1996. 329S: p. S89-S98.

9. Beaulé, P.E., Le Duff, M, Campbell, P, Dorey, F J, Hyun Park, S, Amstutz, H C, Metal-onmetal surface arthroplasty with a cemented femoral component: a 7-10 year follow-up study. J Arthoplasty, 2004. 19(8): p. 17-22.

10. Hing, C.B., Young, D A, Dalziel, R E, Bailey, M, Back, D L, Shimmin, A J, Narrowing of the neck in resurfacing arthroplasty of the hip. J Bone Joint Surg $[\mathrm{Br}], 2007$. 89-B: p. 1019-1024.

11. Spencer, S., Carter, R, Murray, H, Meek, R M D, Femoral Neck Narrowing After Metalon-Metal Hip Resurfacing. J Arthroplasty, 2008. 23: p. 1105-1109.

12. Berend, M.E., Bertrand, T, Metal-Metal Hip Resurfacing: Solution to a Nonexistent Problem. Orthopedics, 2007. 30: p. 724-727.

13. Ong, K.L., Day, J S, Kurtz, S M, Field, R E, Manley, M T,, Role of Surgical Positioning on Interface Stress and Initial Bone Remodelling Stimulus around Hip Resurfacing Arthroplasty. J Arthroplasty, 2008. In Press.

14. Charnley, J., Low Friction Arthroplasty of the Hip: Theory and Practice. 1979, New York: Springer.

15. McGrory, B.J., Morrey, B F, Cahalan, T D, An, K-N, Cabanela, M E, Effect of Femoral Offset on Range of Motion and Abductor Muscle Strength after Total Hip Arthroplasty. J Bone Joint Surg [Br], 1995. 77-B: p. 865-869.

16. Silva, M., Haeng Lee, K, Heisel, C, dela Rosa, M A, Schmalzried, T P, The Biomechanical Results of Total Hip Resurfacing Arthroplasty. J Bone Joint Surg [Am], 2004. 86: p. 40-46.

17. Beaulé, P.E., Lee, J L, Le Duff, M J, Amstutz, H C, Ebramzadeh, E, Orientation of the femoral component in surface arthroplasty of the hip. A biomechanical and clinical analysis. J Bone Joint Surg [Am], 2004. 86: p. 2015-2021.

18. Charnley, J., Anchorage of the femoral head prosthesis to the shaft of the femur. J Bone Joint Surg [Br], 1960. 42-B: p. 28-30.

19. Howie, D.W., Campbell, D, McGee, M, Cornish, B L, Wagner resurfacing hip arthroplasty. The results of one hundred consecutive arthroplasties after eight to ten years. J Bone Joint Surg [Br], 1990. 72: p. 708-714.

20. Amstutz, H.C., Dorey, F, O'Carroll, P F, THARIES resurfacing arthroplasty, evolution and long term results. Clin Ortho Rel Res, 1986. 213: p. 92-139.

21. Ritter, M.A., Lutgring, J D, Berend, M E, Pierson, J L, Failure Mechanisms of Total Hip Resurfacing: Implications for the Present. Clin Orth Rel Res, 2006. 453: p. 110-114. 
22. Taylor, M., Finite element analysis of the resurfaced femoral head. Proc IMechE H, 2006. 220: p. 289-297.

23. Keyak, J.H., Rossi, S A, Jones, K A, Skinner, H B, Prediction of femoral fracture load using automated finite element modelling. J Biomech, 1998. 31: p. 125-133.

24. Keyak, J.H., Rossi, S A, Prediction of femoral fracture load using finite element models: an examination of stress- and strain-based failure theories. J Biomech, 2000. 33: p. 209214.

25. Lotz, J.C., Cheal, E J, Hayes, W C, Fracture Prediction for the Proximal Femur using Finite Element Models: Part I- Linear Analysis. J Biomech Eng, 1991. 113: p. 353-360.

26. Lotz, J.C., Cheal, E J, Hayes, W C, Fracture Prediction for the Proximal Femur using Finite Element Models: Part II- Nonlinear Analysis. J Biomech Eng, 1991. 113: p. 361365.

27. Schileo, E., Taddei, F, Cristolfini, L, Viceconti, M, Subject-specific finite element models implementing a maximum principal strain criterion are able to estimate failure risk and fracture location on human femurs tested in-vitro. J Biomech, 2008. 41: p. 356-367.

28. Bayraktar, H.H., Morgan, E F, Niebur, G L, Morris, G E, Wong, E K, Keaveny, T M, Comparison of the Elastic and Yield Properties of Human Femoral Trabecular and Cortical Bone Tissue. J Biomech, 2004. 37: p. 27-35.

29. Weinans, H., Huiskes, R, Verdonschot, N, van Rietbergen, B, The Effect of Adaptive Bone Remodelling Threshold Levels on Resorption around Noncemented Hip Stems, in Advances in Bioengineering, R. Vanderby, Editor. 1991, ASME: New York. p. 303-306.

30. Weinans, H., Huiskes, R, van Rietbergen, B, Sumner, D R, Turner, T M, Galante, J O, Adaptive bone remodelling around a bonded noncemented total hip arthroplasty: a comparison between animal experiments and computer simulation. J Orth Res, 1993. 11: p. 500-513.

31. Gupta, S., New, A M R, Taylor, M, Bone remodelling inside a cemented resurfaced femoral head. Clin Biomechanics, 2006. 21: p. 594-602.

32. Davis, E.T., Olsen, M, Zdero, R, Waddell, J P, Semlitsch, E H, Femoral Neck Fracture Following Resurfacing. J Bone Joint Surg [Br], 2008. 90-B: p. 1522-1527.

33. Appleyard, R., Walter, W L, Walter, L, Gillies, R M. The Influence of Femoral Head Resurfacing on Femoral Neck Strain: A Biomechanical Study. in 55th Annual Meeting of the Orthopaedic Research Society. 2009. Las Vegas, Nevada.

34. Wagner, H., Surface replacement arthroplasty of the hip. Clin Ortho Rel Res, 1978. 134: p. $102-130$.

35. Amstutz, H.C., Grigoris, P, Dorey, F J, Evolution and future of surface replacement of the hip. J Ortho Sci, 1998. 3: p. 169-186.

36. Freeman, M.A.R., Cameron, H U, Brown, G C, Cemented double cup arthroplasty of the hip. Clin Ortho Rel Res, 1978. 134(45-52).

37. Huiskes, R., Chao, E Y S, A Survey of Finite Element Analysis in Orthopedic Biomechanics: the First Decade. J Biomech, 1983. 16: p. 385-409.

38. de Waal Malefijt, M.C., Huiskes, R, A clinical, radiological and biomechanical study of the TARA hip prosthesis. Acta Orthop Trauma Surg, 1993. 112: p. 220-225.

39. Wanatabe, Y., Shiba, N, Matsuo, S, Higuchi, F, Tagawa, Y, Inoue, A, Biomechanical study of the resurfacing hip arthroplasty- finite element analysis of the femoral component. J Arthoplasty, 2000. 15: p. 505-511.

40. Little, J.P., Taddei, F, Viceconti, M, Murray, D W, Gill, H S, Changes in Femur Stress after Hip Resurfacing Arthroplasty: Response to Physiological Loads. Clin Biomechanics, 2007. 22: p. 440-448.

41. Ong, K.L., Kurtz, S M, Manley, M T, Rushton, N, Mohammed, N A, Field, R E, Biomechanics of the birmingham hip arthroplasty. J Bone Joint Surg [Br], 2006. 88-B: p. 1110-1115.

42. Long, J., Bartel, DL, Surgical Variables Affect Mechanics of a Hip Resurfacing System. Clin Ortho Rel Res, 2006. 453: p. 115-122.

43. Radcliffe, I.A.J., Taylor, M, Investigation into the Effect of Varus-Valgus Orientation on Load Transfer in the Resurfaced Femoral Head: a Multi-Femur Finite Element Analysis. Clin Biomechanics, 2007. 22: p. 780-786. 
44. Radcliffe, I.A.J., Taylor, M, Investigation into the Affect of Cementing Techniques on Load Transfer in the Resurfaced Femoral Head: A Multi-Femur Finite Element Analysis. Clin Biomechanics, 2007. 22: p. 422-430.

45. Long, J., Santner, TJ, Bartel, DL, Hip Resurfacing Increases Bone Strains Associated with Short-Term Femoral Neck Fracture. J Orth Res, 2009: p. 1-7.

46. Steffen, R.T., Pandit, H P, Palan, J, Beard, D J, Gundle, R, McLardy-Smith, P, Murray, D W, Gill, H S, The five-year results of the Birmingham Hip Resurfacing Arthroplasty. J Bone Joint Surg [Br], 2008. 90-B: p. 436-441. 


\section{FIGURE CAPTIONS}

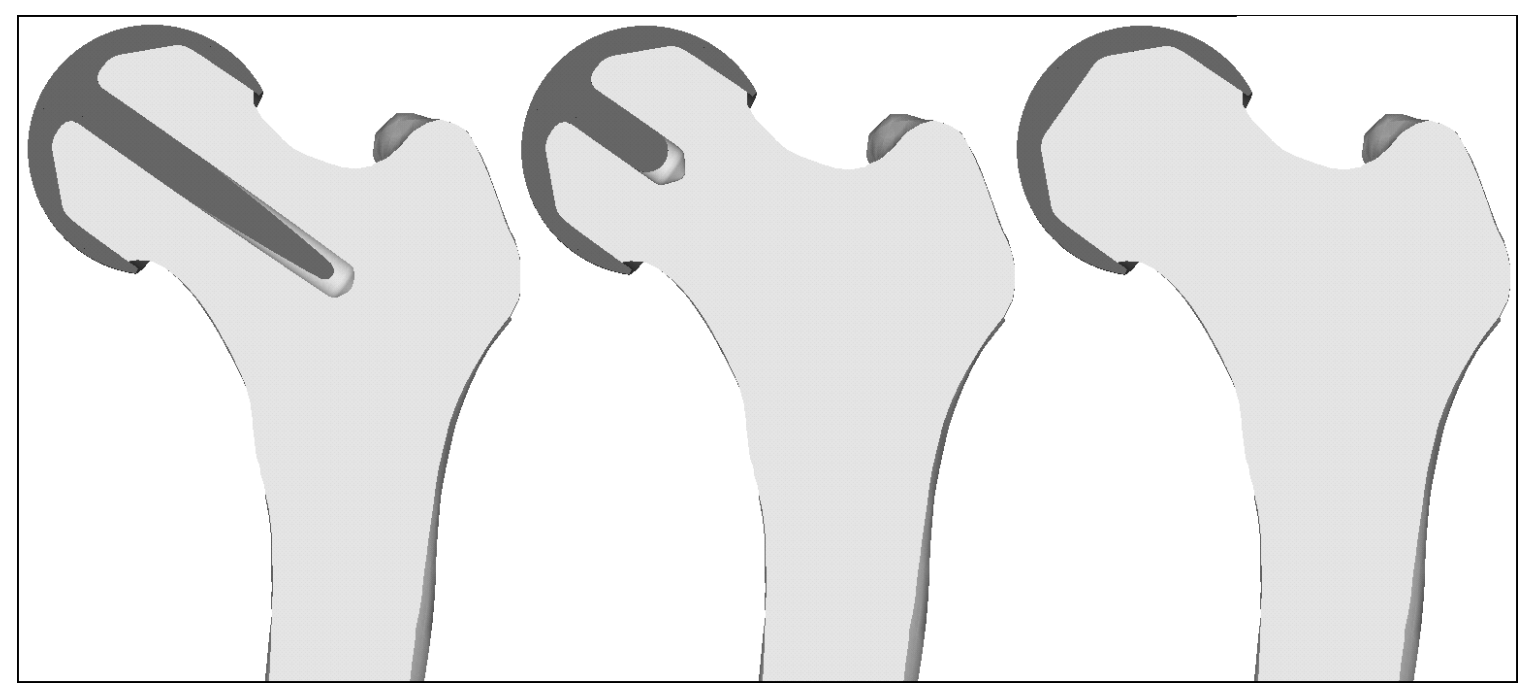

Fig. 1: The Three Prosthesis Designs Investigated: Traditional (left), Stemless (centre) and Short

\section{Stemmed (right)}

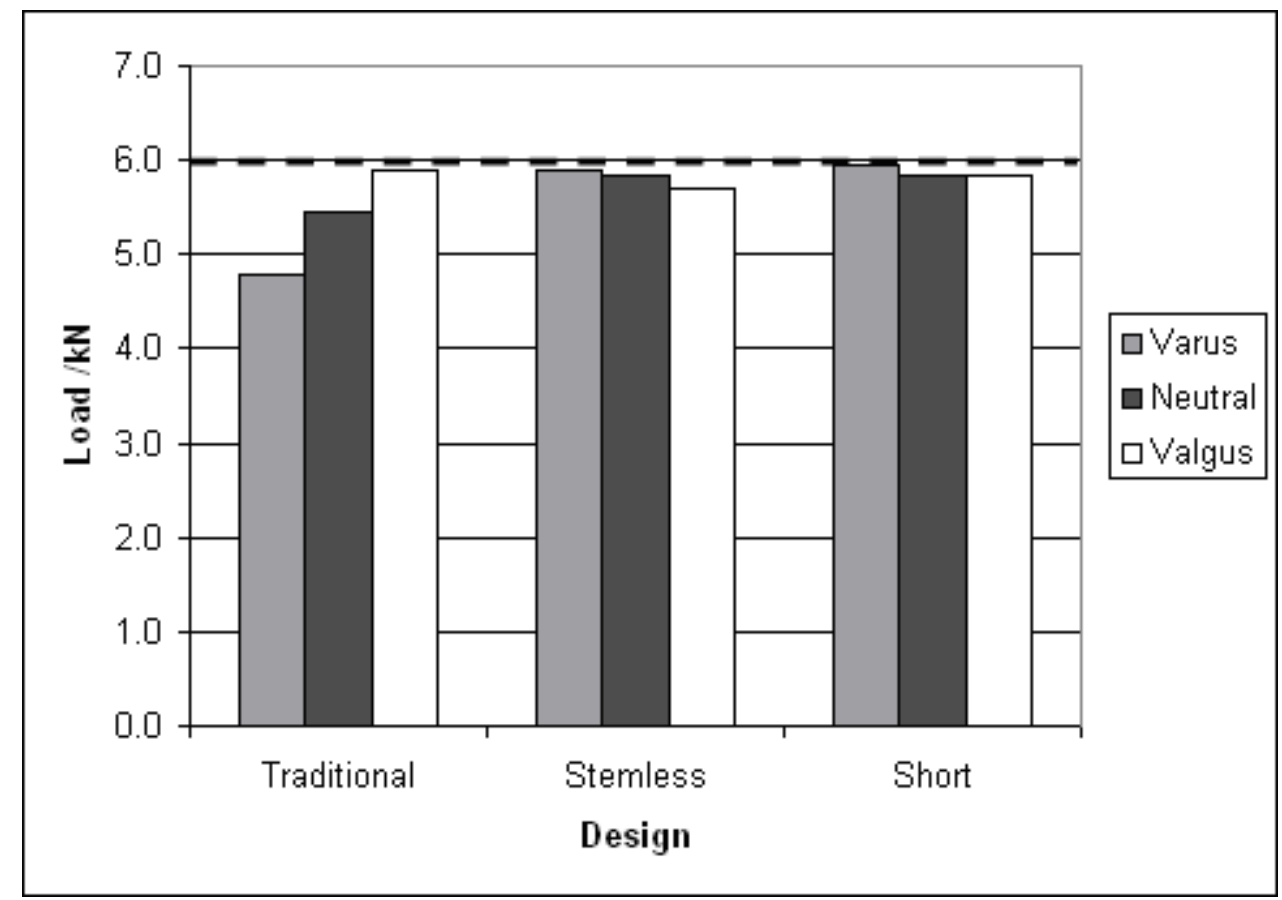

Fig. 2: Predicted Femoral Neck Fracture Load under Stumbling Conditions, for the Femur

Resurfaced with each of the Three Resurfacing Head Designs in Varus, Neutral and Valgus

Orientations. Dashed Line Marks Fracture Load for Intact Bone 


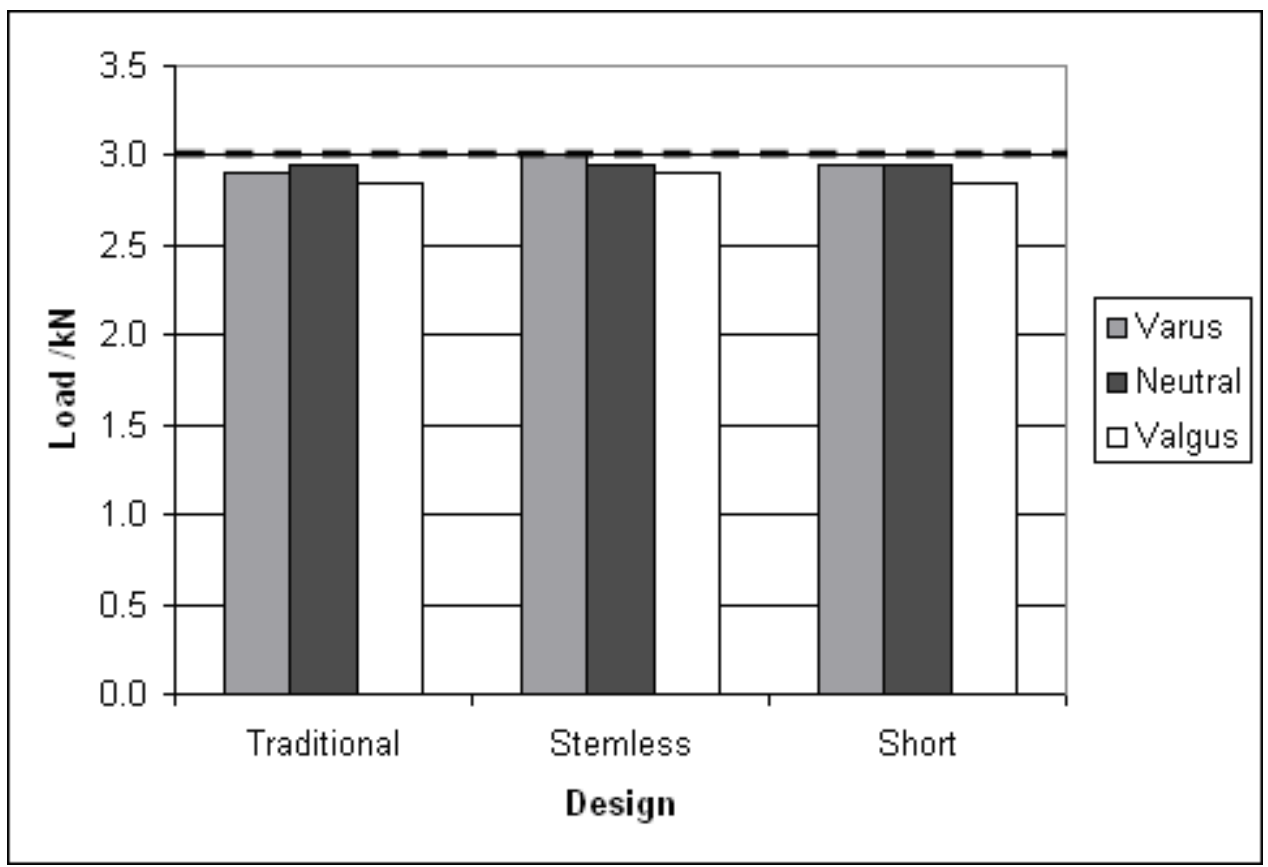

Fig. 3: Predicted Femoral Neck Fracture Load under Sideways Fall Conditions, for the Femur Resurfaced with each of the Three Resurfacing Head Designs in Varus, Neutral and Valgus Orientations. Dashed Line Marks Fracture Load for Intact Bone 


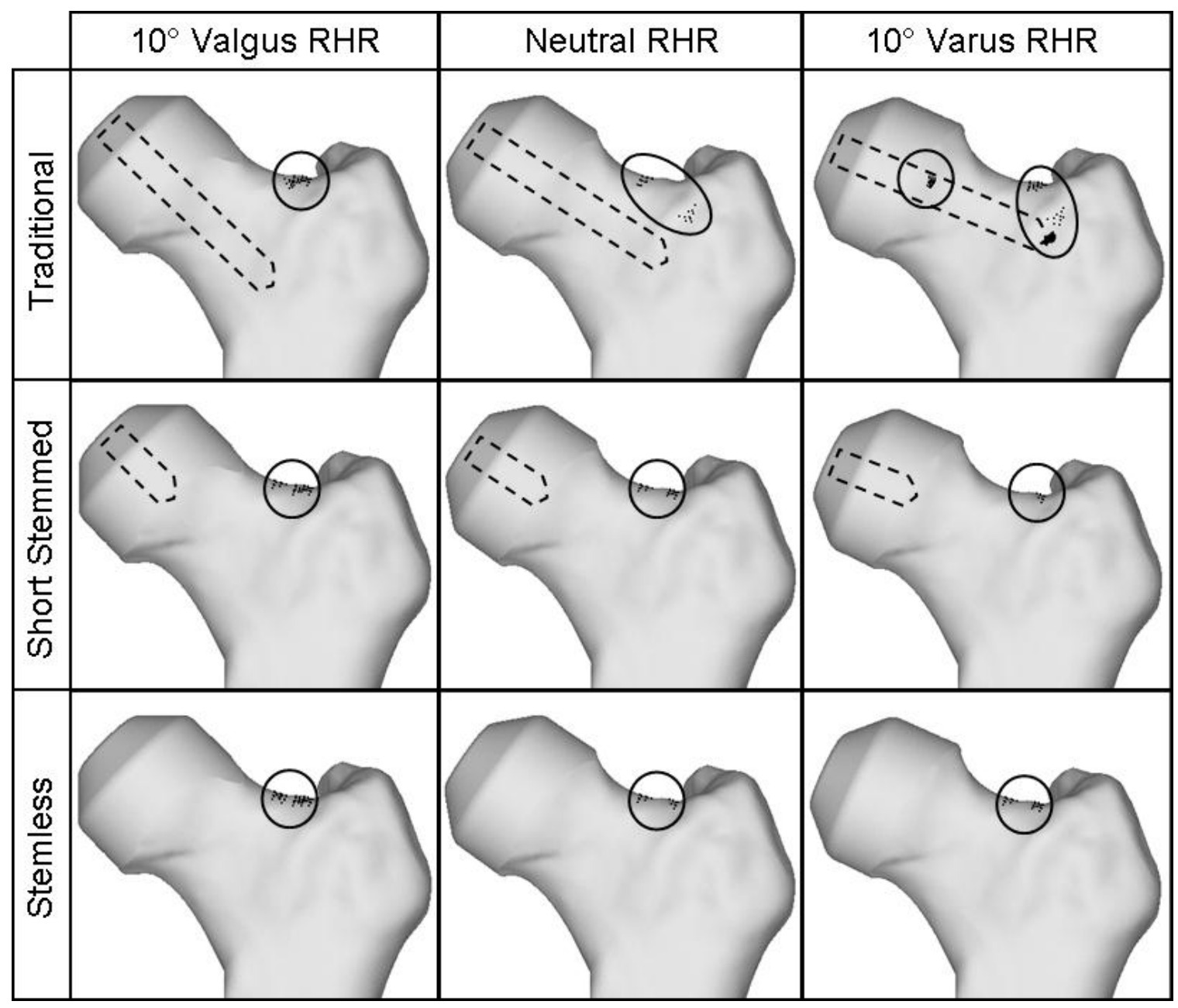

Fig. 4: Distribution of Yielding Bone Elements under $6 \mathrm{kN}$ Stumbling Load, for the Femur

Resurfaced with the Three Prosthesis Designs in Valgus, Neutral and Varus Orientation. 


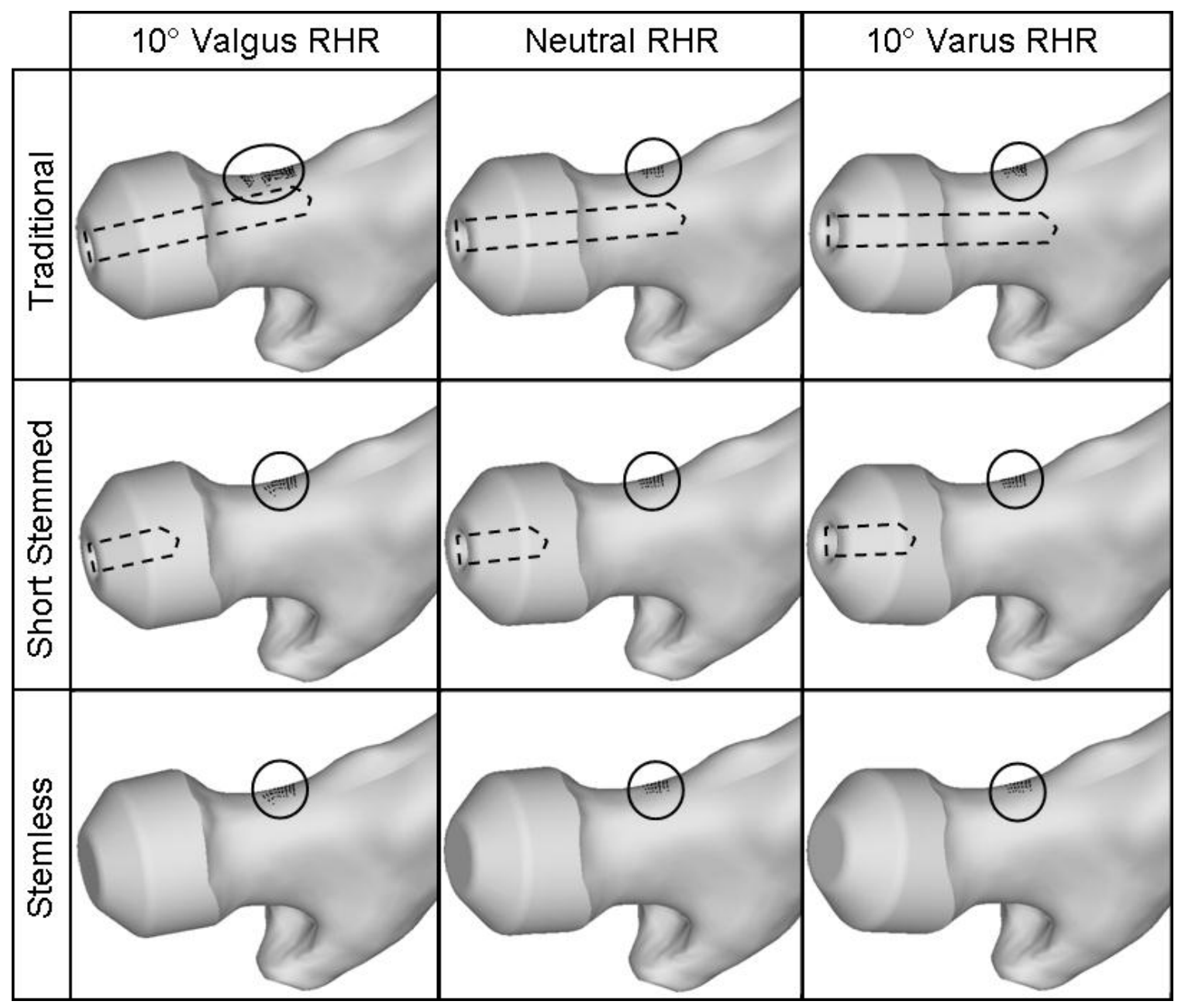

Fig. 5: Distribution of Yielding Bone Elements under 3kN Falling Load, for the Femur Resurfaced with the Three Prosthesis Designs in Valgus, Neutral and Varus Orientation. 


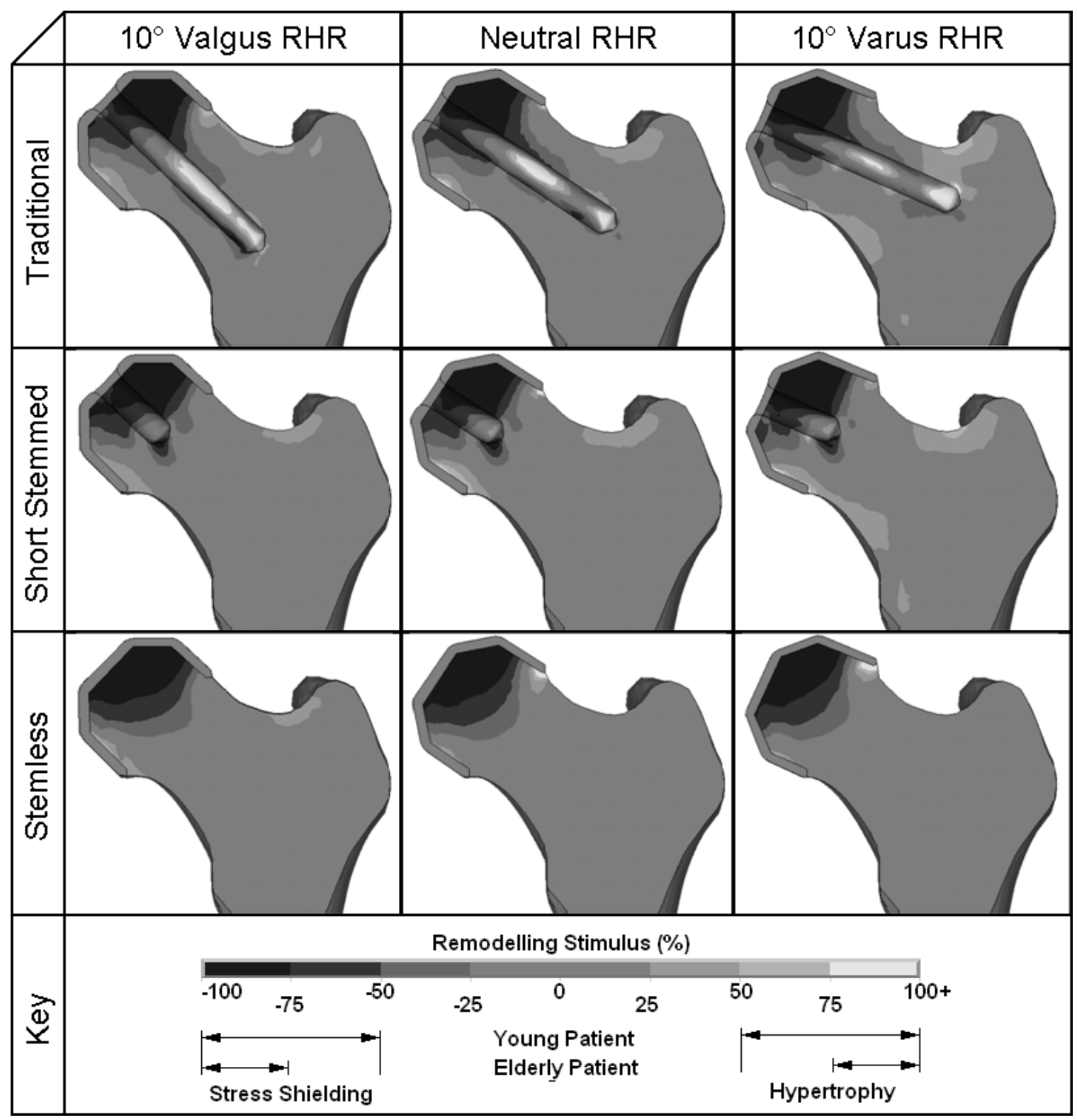

Fig. 6: Strain Energy Density Remodelling Stimulus for Resurfacing with the Three Prosthesis Designs in Valgus (left), Neutral (middle) and Varus (right) Orientations. 


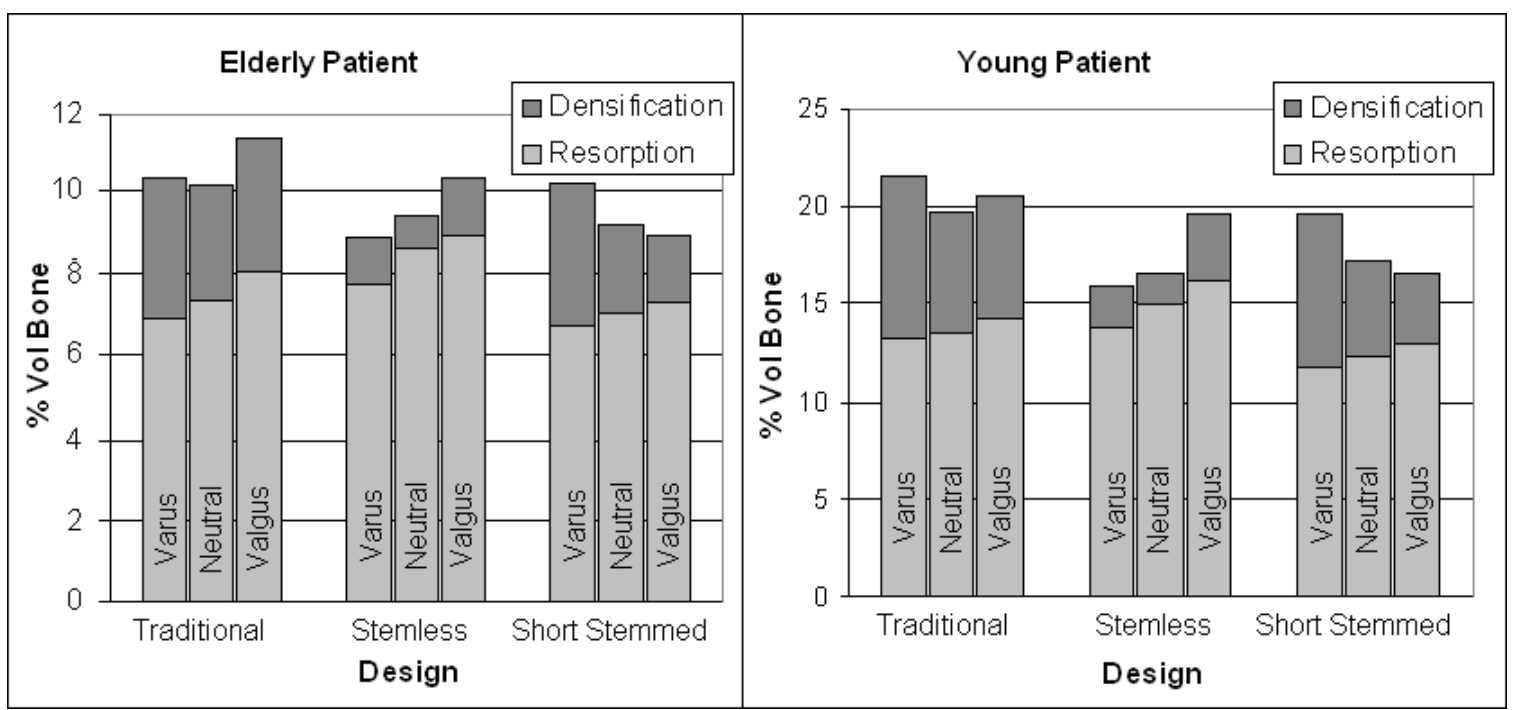

Fig. 7: Percentage Volumes of Remodelling Femoral Head and Neck Bone, Resurfaced with the Three Prosthesis Designs, in Elderly (left) and Young (right) Patients. 